\title{
Anti-PD-1 Monoclonal Antibody MGA012
}

National Cancer Institute

\section{Source}

National Cancer Institute. Anti-PD-1 Monoclonal Antibody MGA012. NCI Thesaurus.

Code C142168.

A proprietary humanized monoclonal antibody directed against the negative immunoregulatory human cell surface receptor programmed cell death 1 (PD-1), with potential immune checkpoint inhibitory and antineoplastic activities. Upon administration, anti-PD-1 monoclonal antibody MGA012 binds to and inhibits PD-1 and its downstream signaling pathways. This may restore immune function through the activation of T-cells and cell-mediated immune responses against tumor cells. PD-1, a transmembrane protein in the immunoglobulin superfamily (IgSF) expressed on T-cells, functions as an immune checkpoint that negatively regulates $\mathrm{T}$-cell activation and effector function when activated by its lig ands programmed cell death ligand 1 (PD-L1) or 2 (PD-L2); it plays an important role in tumor evasion from host immunity. 